\title{
Intravitreal Bevacizumab with Grid Photocoagulation for Recurrent Macular Edema Secondary to Retinal Vein Occlusion
}

\author{
Salah M. Mady ${ }^{1}$, Asaad A. Ghanem ${ }^{2 *}$ \\ ${ }^{1}$ Department of Ophthalmology, Faculty of Medicine, Banha University, Banha, Egypt; ${ }^{2}$ Mansoura Ophthalmic Center, Faculty of \\ Medicine, Mansoura University, Mansoura, Egypt. \\ Email: a asaadghanem@hotmail.com
}

Received December $7^{\text {th }}, 2013$; revised January $8^{\text {th }}, 2014$; accepted January $11^{\text {th }}, 2014$

Copyright (C) 2014 Salah M. Mady, Asaad A. Ghanem. This is an open access article distributed under the Creative Commons Attribution License, which permits unrestricted use, distribution, and reproduction in any medium, provided the original work is properly cited. In accordance of the Creative Commons Attribution License all Copyrights (C 2014 are reserved for SCIRP and the owner of the intellectual property Salah M. Mady, Asaad A. Ghanem. All Copyright @ 2014 are guarded by law and by SCIRP as a guardian.

\begin{abstract}
Purpose: To assess the efficacy of intravitreal bevacizumab (IVB) combined with grid photocoagulation in the management of recurrent macular edema secondary to retinal vein occlusion (RVO). Methods: This is a prospective, non-randomized, interventional study. Thirty five eyes with branch retinal vein occlusion (BRVO) and 15 eyes with central retinal vein occlusion (CRVO) were treated with grid photocoagulation combined with IVB for recurrent macular edema. The visual acuity, central macular thickness and intraocular pressure were outcome measures. The mean duration of follow-up was $18.1 \pm 3.6$ months. Results: One month after treatment, 45 of the 50 eyes showed complete resolution of the cystoid space. Compared with initial values, final central macular thickness was reduced significantly in both BRVO and CRVO groups $(P<0.001)$, but improvement in VA was significant only for eyes with BRVO $(P=0.012)$. The total number of IVB was $1.8 \pm 0.3$ for eyes with either BRVO or CRVO. Conclusion: IVB combined with grid photocoagulation is an effective treatment for reducing recurrent macular edema associated with RVO.
\end{abstract}

\section{KEYWORDS}

Bevacizumab; Grid Laser Photocoagulation; Macular Edema; OCT; RVO

\section{Introduction}

Macular edema remains one of the most frequent cause of visual impairment associated with retinal vein occlusion (RVO). Increased intravascular pressure and reduced blood flow in macular capillaries can lead to dysfunction of the endothelial blood-retinal barrier, increased vascular permeability and leakage from the retinal capillaries, which results in macular edema [1-3]. Vascular endothelial growth factor (VEGF), which is a potent inducer of increased vascular permeability that causes leakage from retinal vessels and contributes to macular oedema [4].

Because of the promising short-term effects of intravitreal bevacizumab (IVB), which is a recombinant human

\footnotetext{
${ }^{*}$ Corresponding author.
}

monoclonal antibody that binds to VEGF, anti-VEGF therapy is becoming a primary treatment for this condition [5]. Noma et al. [6] reported that increased intraocular levels of VEGF and sICAM-1 or a decreased level of PEDF are associated with increased vascular permeability and the severity of retinal ischemia in BRVO patients.

Laser and steroid treatment for patients with RVO have had statistically significant benefits in terms of retinal thickening and visual acuity, but with several limitations [7].

Grid photocoagulation is an evidence-based treatment of the macular edema associated with RVO. In a report by the Branch Vein Occlusion Study Group, [8] grid photocoagulation to macular edema caused by branch retinal vein occlusion (BRVO) significantly improved long-term visual prognosis. 
In the present study, we evaluate both visual function (visual acuity) and retinal morphology (central macular thickness) in RVO patients using grid photocoagulation in combination with IVB for recurrent macular edema.

\section{Patients and Methods}

\subsection{Study Design}

This was a prospective, non-randomized, interventional study. After explaining the details of the study, we obtained written informed consent from all patients before enrollment. The study was approved by El-Nour center for eye surgery and LASIK, trust ethics committee and was carried out in accordance with the Declaration of Helsinki (1989) of the world medical association.

\subsection{Patients}

Thirty five patients (35 eyes) with BRVO and 15 patients (15 eyes) with CRVO were included in the study. A recurrence of macular edema was defined as a relapse of the cystoid space at the foveal center and an increase of the foveal thickness to more than $300 \mu \mathrm{m}$. Previous treatment for macular edema included 29 eyes had been treated with grid laser photocoagulation, and 21 eyes had received IVT.

Exclusion criteria included: 1) history of glaucoma or ocular hypertension, 2) previous intraocular surgery or intravitreal injection of triamcinolone acetnoide, 3) prior vitrectomy, 4) eyes with co-existing epiretinal membrane or serous retinal detachment, and 5) significant media opacity.

Patients were diagnosed as having hypertension if their systolic blood pressure was $\geq 140 \mathrm{mmHg}$ and diastolic blood pressure was $>90 \mathrm{mmHg}$, or if the patient was already taking antihypertensive medication. A diagnosis of hyperlipidemia was based on a total cholesterol $\geq 240$ $\mathrm{mg} / \mathrm{dL}$, triglycerides $\geq 160 \mathrm{mg} / \mathrm{dL}$, low-density lipoprotein cholesterol $\geq 130 \mathrm{mg} / \mathrm{dL}$, or use of cholesterol lowering medication [9].

Complete ocular examination was done at each visit in form of best-corrected visual acuity (BCVA), measurement of intraocular pressure (IOP) using Goldmann applanation tonometry, funduscopy, slit-lamp biomicroscopy with a Goldmann three mirror contact lens, and central macular thickness (CMT) measurement using optical coherence tomography (Zeiss Stratus OCT 3, Carl Zeiss Meditec, Dublin, CA). In addition, patients underwent standard fundus color photography and fundus fluorescein angiography, which was performed with a Topcon TRC-50EX fundus camera, an image-net system (Tokyo Optical Co. Ltd., Japan), and a preset lens with a slit-lamp.
CMT was calculated as the average retinal thickness within a circle having a $500 \mu \mathrm{m}$ radius centered on the fovea. Retinal thickness was defined as the distance between the inner surface of the neurosensory retina and the retinal pigment epithelium.

Initially, 35 eyes underwent one IVB injection, and 15 eyes underwent two IVBs injections. At the time of initial IVB, CMT was greater than $300 \mu \mathrm{m}$ and visual acuity was equal to or less than 0.7 on a Landolt chart.

Intravitreal injections were performed under complete sterile conditions using bovidine iodine $10 \%$ for eye lid skin and $5 \%$ for ocular surface wash. After complete draping, surface anesthesia was applied. Intravitreal injection was performed as follows: $1.25 \mathrm{mg}$ bevacizumab in $0.05 \mathrm{~mL}$ (Avastin; Gemetech Inc., San Fransisco, CA) was injected in the superotemporal quadrant using 27gauge needle, a needleless syringe was pressed against the globe. To avoid an increase of IOP, aqueous humor paracentesis was removed as appropriate. Topical mixed antibiotics and steroids (tobramycin $0.3 \%$ and dexamethazone $0.1 \%$ ) were applied for 1 week, and topical bblocker was used to control intraocular pressure.

For the studied patients, grid photocoagulation was performed immediately after the macular edema had been reduced by an IVB. Grid photocoagulation to the leaking area seen on the fluorescein angiogram was performed with a multicolor diode laser (MC-300; Nidek, Gamagori, Japan) with settings of $561 \mathrm{~nm}$ wavelength and $100 \mu \mathrm{m}$ spot size for 0.1 second; sufficient power was used to produce a burn detectable at the level of the retinal pigment epithelium. Examination of BCVA, IOP, detection of anterior chamber reaction, funduscopy and detection of CMT were performed at first day, and weeks 1, 6, 12 after injection. All patients had at least a 12month follow-up after the combination therapy; the mean duration of total follow-up time was $18.1 \pm 3.6$ months.

\subsection{Statistical Analysis}

Statistical analysis was performed using version 17.0 (SPSS, Chicago, IL). All results are presented as mean \pm standard deviation or frequency. Best-corrected visual acuity was measured with a Landolt chart and converted to a logarithm of the minimum angle of resolution (logMAR). Visual acuity and CMT were compared with Tukey's HSD test after one factor repeated measurement of analysis of variance. Pearson's correlation coefficients were calculated. A two-tailed $P$ value of less than 0.05 was considered to be statistically significant.

\section{Results}

Table 1 shows demographic and clinical characteristics 
Table 1. Demographic and clinical characteristic of the studied patients.

\begin{tabular}{|c|c|c|}
\hline & BRVO & CRVO \\
\hline Number of eyes & 35 & 15 \\
\hline Gender (male/female) & $11 / 24$ & $8 / 7$ \\
\hline Age (years) & $62.3 \pm 5.2$ & $62.1 \pm 4.3$ \\
\hline Hypertension & 18 & 9 \\
\hline Systolic blood pressure (mmHg) & $136 \pm 13$ & $135 \pm 15$ \\
\hline Diastolic blood pressure (mmHg) & $85 \pm 12$ & $86 \pm 11$ \\
\hline Hyperlipidemia & 20 & 8 \\
\hline Diabetes mellitus & 16 & 6 \\
\hline \multicolumn{3}{|l|}{ Before IVB injection } \\
\hline Visual acuity (logMAR) & $0.63 \pm 0.21$ & $0.89 \pm 0.31$ \\
\hline Central macular thickness $(\mu \mathrm{m})$ & $625.1 \pm 163.2$ & $753.3 \pm 134.2$ \\
\hline Number of IVB (once/twice) & $11 / 6$ & $7 / 5$ \\
\hline \multicolumn{3}{|l|}{ Baseline (before combined therapy) } \\
\hline Visual acuity (logMAR) & $0.54 \pm 0.16$ & $0.73 \pm 0.24$ \\
\hline Central macular thickness $(\mu \mathrm{m})$ & $514.2 \pm 124.1$ & $583.6 \pm 126.2$ \\
\hline Duration between initial IVB and baseline (months) & $7.6 \pm 3.5$ & $4.7 \pm 1.2$ \\
\hline Follow-up (months) & $18.0 \pm 6.1$ & $16.1 \pm 4.2$ \\
\hline
\end{tabular}

$\mathrm{BRVO}=$ branch retinal vein occlusion; $\mathrm{CRVO}=$ central retinal vein occlusion; IVB = intravitreal injection of bevacizumab, logMAR = logarithm of the minimum angle of resolution.

of the patients included in the study. Before initial IVBs injection, all eyes showed marked increase in central macular thickness (CMT) associated with RVO, often with a foveal cystoid space. Immediately after injection, a reduction in CMT was seen and confirmed by OCT. Compared with pre-injection measurements (625.1 \pm $163.2 \mu \mathrm{m}$ in the BRVO group and $753.3 \pm 134.2 \mu \mathrm{m}$ in the CRVO group), CMT was decreased significantly to 225.2 $\pm 112.5 \mu \mathrm{m}$ in eyes with BRVO $(P<0.001)$ and to $235.8 \pm$ $122.1 \mu \mathrm{m}$ in eyes with CRVO $(P<0.001)$ (Figure 1$)$. In parallel to the reduction in macular thickness, mean VA improved from $0.63 \pm 0.21$ to $0.36 \pm 0.28$ for eyes with BRVO $(P<0.001)$, and from $0.89 \pm 0.31$ to $0.65 \pm 0.21$ for eyes with CRVO $(P=0.071)$ (Figure 2).

Just before grid photocoagulation combined with IVB therapy, all eyes showed cystoid spaces beneath the fovea, and the mean CMT had increased to $514.2 \pm 124.1$ $\mu \mathrm{m}$ in the BRVO group and to $583.6 \pm 126.2 \mu \mathrm{m}$ in the CRVO group. The elapsed times from the previous IVB to the current combined therapy was $6.1 \pm 2.6$ months for eyes with BRVO and $4.5 \pm 1.5$ months for eyes with CRVO. The improvement in VA by the previous IVBs had receded at the baseline visit; mean VA was now 0.54 \pm 0.16 in the BRVO group and $0.73 \pm 0.24$ in the CRVO group.

On the combination therapy, IVB done first. Grid photocoagulation was performed after the CMT had cleared at $11.3 \pm 9.4$ days after IVB in eyes with BRVO and at $12.2 \pm 13.2$ days after IVB in eyes with CRVO. With this combination therapy, CMT was reduced significantly to $245.5 \pm 91.3 \mu \mathrm{m}$ in eyes with BRVO $(P<$ $0.001)$ and to $252.2 \pm 51.0 \mu \mathrm{m}$ in eyes with CRVO $(P<$ $0.001)$ at one month, and VA improved slightly to $0.45 \pm$ 0.10 in eyes with BRVO $(P=0.632)$, and to $0.73 \pm 0.10$ in eyes with CRVO $(P=0.623)$.

One month after treatment, 31 of the 35 eyes with BRVO and 12 of the 15 eyes with CRVO showed complete resolution of the cystoid space (Figure 3). However, the cystoid space reappeared, to at least some extent, in 23 eyes with BRVO and in 9 eyes with CRVO (Figure 4). Mean duration from the combination therapy to the recurrence was $3.1 \pm 1.6$ months in eyes with BRVO and $4.1 \pm 0.4$ months in eyes with CRVO. Fifteen eyes with BRVO and 3 eyes with CRVO needed additional IVB or grid photocoagulation, so the total number of IVB was $2.4 \pm 0.3$ for both BRVO and CRVO.

Mean follow-up after this therapy was $18.0 \pm 4.1$ months in BRVO eyes and $20.1 \pm 2.3$ months in eyes with CRVO. Comparing both VA and CMT just before the initial IVB, final CMT was reduced significantly in both BRVO eyes and CRVO eyes $(P<0.001$, respectively), but the improvement in VA was significant only in those eyes with BRVO $(P=0.014)$.

\section{Discussion}

Intravitreal bevacizumab injection showed a reduction of 


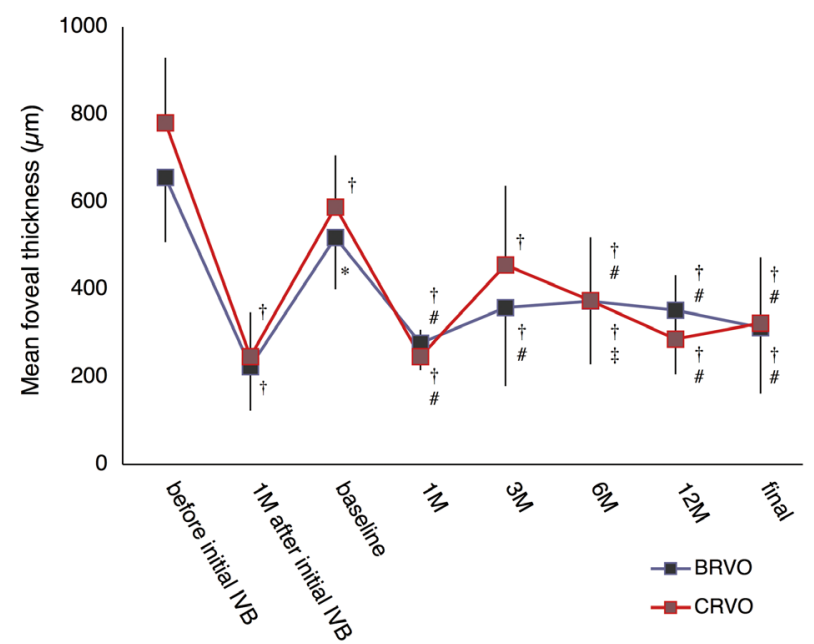

Figure 1. Mean foveal thickness in eyes that were treated with grid photocoagulation combined with an IVB for the treatment of recurrent macular edema associated with RVO. Compared with values before the initial treatment; compared with baseline values (before combined grid photocoagulation and IVB). $M=\operatorname{month}(s)(P<0.05 ; P<0.01)$.

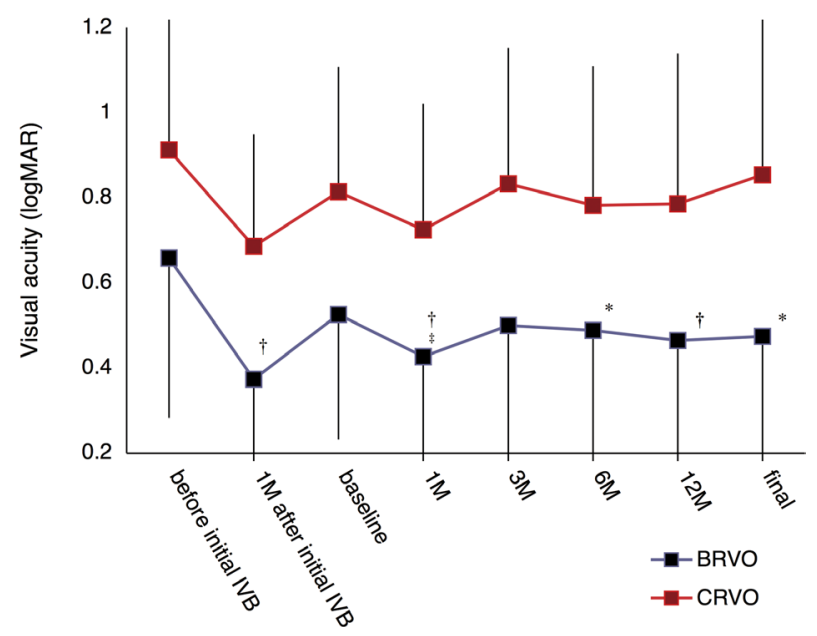

Figure 2. Visual acuity in logMAR of eyes treated with grid photocoagulation combined with IVB for the treatment of recurrent macular edema associated with RVO. Compared with values before the initial treatment, compared with baseline values (before combined grid photocoagulation and IVB). $\mathrm{M}=\operatorname{month}(\mathrm{s})(P<0.05 ; P<0.01)$.

macular oedema soon after the injection. Measurement of visual acuity and measurement of retinal thickness by optical coherence tomography are widely considered to be useful for determining the treatment strategy in patients with RVO.

In a collaborative retina study, the mean number of $1.25 \mathrm{mg}$ IVB injections over a 24-month period was 7.2 for the treatment of macular edema associated with CRVO [10]. Recently, however, large randomized clinical trials have revealed the 6-month results of monthly

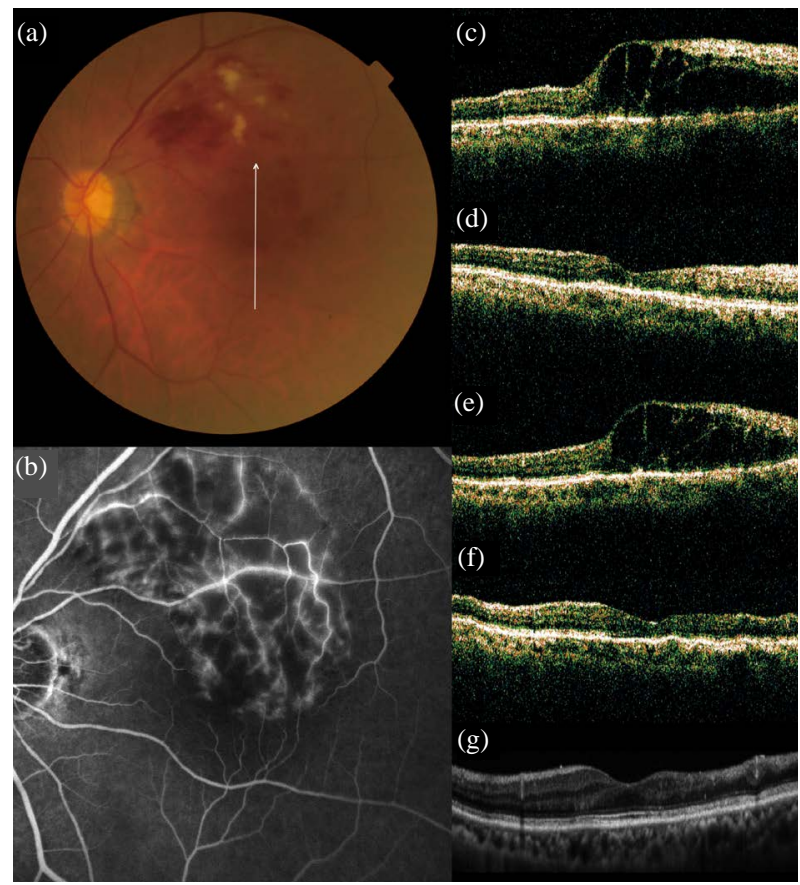

Figure 3. No recurrence of macular edema after grid photocoagulation combined with intravitreal bevacizumab (IVB) for recurrent macular edema associated with BRVO. (a) An 75-year-old woman had a visual disturbance in the left eye due to macular edema associated with BRVO; (b) Fluorescein angiogram shows fluorescein leakage from the capillaries; (c)-(g) Vertical sectional images centered on the fovea with OCT were obtained at the initial visit (c), at 1 month after the initial IVB (d), just before the combined therapy at baseline (e), at 1 month after combined therapy (f), and at 3 the final visit (g).

injections of ranibizumab for RVO [11]. With monthly injections for a 6 month period, retinal thickening was continuously resolved and more than $40 \%$ of eyes with CRVO and more than $50 \%$ of eyes with BRVO gained 15 letters on VA testing, and a VA of equal to or better than 20/40 was achieved in $40 \%$ of eyes with CRVO and in $60 \%$ of eyes with BRVO. Based on these reports, frequent injection of this anti-VEGF agent seems to be promising for macular edema due to RVO.

To reduce the number of injections, 1 ) it would be effective to distinguish those patients who do not require re-treatment [12], 2) to design the optimum dosing and sequence [13], or 3) to switch to some other treatment if anti-VEGF therapy is not effective.

In the present study, we aimed to reduce the number of IVB in eyes with recurrent macular edema by co-performing grid photocoagulation. Since the Branch Vein Occlusion Study Group recommended grid photocoagulation for macular edema associated with BRVO, for 25 years this has been seen as the primary treatment. The Central Vein Occlusion Study Group reported that grid 


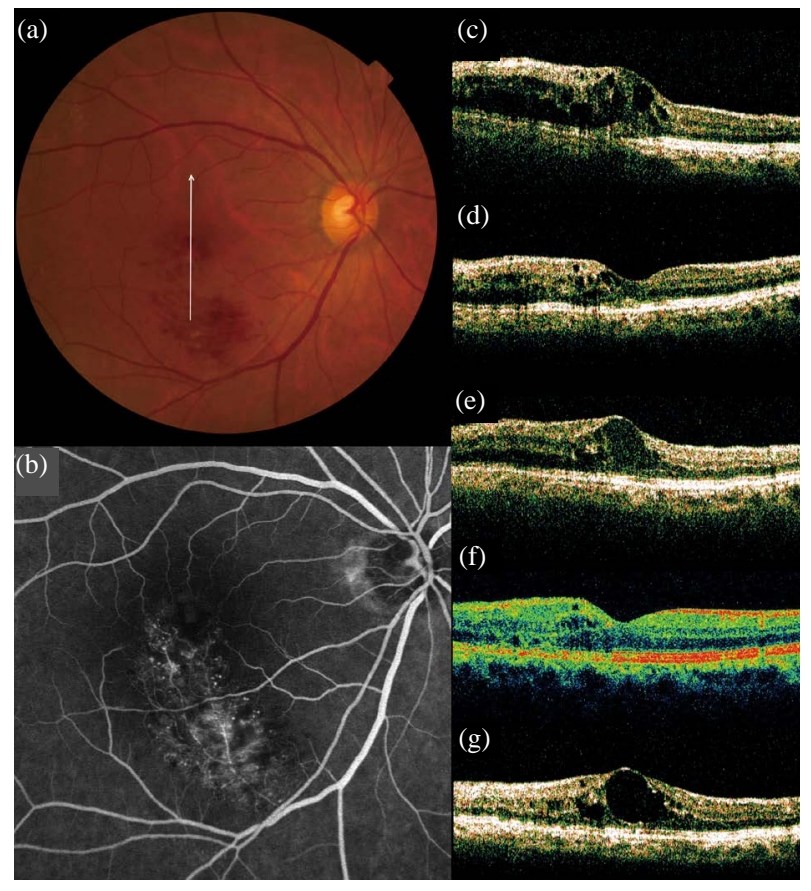

Figure 4. Recurrence of macular edema after grid photocoagulation combined with IVB for recurrent macular edema associated with BRVO. (a) A 72-year-old woman had a visual disturbance in the right eye due to macular edema associated with BRVO; (b) Fluorescein angiogram shows fluorescein leakage from the capillaries; (c)-(g) Vertical sectional images centered on the fovea with OCT were obtained at the initial visit (c), at 1 month after initial IVB (d), just before the combined therapy at baseline (e), at 1 month (f) and at 3 months (g) after the combined therapy.

photocoagulation reduced angiographic macular edema in eyes with CRVO [1].

Macular thickness and volume within the $20^{\circ}$ field were significantly correlated with the nonperfused area. Interestingly, macular sensitivity and macular thickness/ volume within $20^{\circ}$ field showed parallel changes [14].

Tyrberg et al. [15] concluded that alterations of neuronal function in the macular region due to ischemia might precede the deterioration of visual acuity because the ischemic area was correlated with prolongation of the implicit time regardless of whether visual acuity was preserved ( 0.6 or better). Thus, deterioration of visual acuity might occur after alterations of neuronal function due to ischemia.

As macular edema increases, ischemia also worsen, with the result that macular function may be impaired. This hypothesis is supported by previous reports that the retinal thickness at the central fovea influences visual function in RVO patients [16,17]. In addition, tissue expansion due to edema could stretch the capillary network and lead to transient capillary closure because of the high tissue fluid pressure in patients with ischemic macular edema [18].

In the present study, the combination therapy was performed to macular edema recurred after the effective previous IVB. This combination treatment reduced CMT as effectively as did the previous IVB. However, improvement in VA was not as effective as was the previous IVB. Cystoid edema had resolved completely in $87 \%$ of eyes at one month, but $62 \%-75 \%$ of eyes ultimately showed a recurrence. At the final visit, although CMT was significantly reduced compared with baseline values, improvement in VA had receded and had returned to baseline values.

Compared with measurements just before the initial IVB, final CMT was reduced significantly in both the BRVO and CRVO groups, but the improvement in VA was significant only in eyes with BRVO. The present study revealed that grid photocoagulation has substantial effect of reducing the CMT associated with RVO, but its efficacy for visual recovery appears to be limited.

Based on the present results, we concluded that both grid photocoagulation combined with IVB effective for recurrent macular edema associated with RVO.

\section{Declaration of Interest}

None of the authors has a financial or proprietary in any material or method mentioned. The authors alone are responsible for the content of the paper.

\section{Acknowledgements}

The authors thank Taha Baker for his care and diligence during writing the paper.

\section{REFERENCES}

[1] C. Pelzek and J. I. Lim, "Diabetic Macular Oedema: Review and Update," Ophthalmology Clinics of North America, Vol. 15, No. 4, 2002, pp. 555-563. http://dx.doi.org/10.1016/S0896-1549(02)00043-3

[2] J. M. Lahey, D. S. Fong and J. Kearney, "Intravitreal Tissue Plasminogen Activator for Acute Central Retinal Vein Occlusion," Ophthalmic Surgery, Lasers and Imaging, Vol. 30, No. 6, 1999, pp. 427-434.

[3] E. M. Opremcak, R. A. Bruce, M. D. Lomeo, et al., "Radial Optic Neurotomy for Central Retinal Vein Occlusion: A Retrospective Pilot Study of 11 Consecutive Cases,” Retina, Vol. 21, No. 5, 2001, pp. 408-415. http://dx.doi.org/10.1097/00006982-200110000-00002

[4] H. Noma, H. Funatsu, M. Yamasaki, et al., “Aqueous Humor Levels of Cytokines Are Correlated to Vitreous Levels and Severity of Macular Oedema in Branch Retinal Vein Occlusion,” Eye, Vol. 22, No. 1, 2008, pp. 42-48. http://dx.doi.org/10.1038/sj.eye.6702498

[5] J. B. Jonas, I. Kreissig and R. F. Degenring, "Intravitreal Triamcinolone Acetonide as Treatment of Macular Ede- 
ma in Central Retinal Vein Occlusion," Graefe's Archive for Clinical and Experimental Ophthalmology, Vol. 240, No. 9, 2002, pp. 782-783.

http://dx.doi.org/10.1007/s00417-002-0529-0

[6] H. Noma, H. Funatsu, T. Mimura, et al., "Pigment Epithelialum-Derived Factor, Vascular Endothelial Growth Factor in Branch Retinal Vein Occlusion with Macular Edema," Graefe's Archive for Clinical and Experimental Ophthalmology, Vol. 248, No. 11, 2010, pp. 1559-1565. http://dx.doi.org/10.1007/s00417-010-1486-7

[7] D. B. Roth, C. Cukras, R. Radhakrishnan, et al., "Intravitreal Triamcinolone Acetnoide Injections in Treatment of Retinal Vein Occlusions," Ophthalmic Surgery, Lasers and Imaging, Vol. 39, No. 6, 2008, pp. 446-454. http://dx.doi.org/10.3928/15428877-20081101-16

[8] The Branch Vein Occlusion Study Group, “Argon Laser Photocoagulation for Macular Edema in Branch Vein Occlusion," American Journal of Ophthalmology, Vol. 98, No. 3, 1984, pp. 271-282.

[9] A. C. Kirchhoff, M. L. Drum, J. X. Zhang, et al., "Hypertension and Hyperlipidemia Management in Patients Treated at Community Health Centers," Journal of Clinical Outcomes Management, Vol. 15, No. 3, 2008, pp. 125-131.

[10] L. Wu, J. F. Arevalo, M. H. Berrocal, et al., "Comparison of Two Doses of Intravitreal Bevacizumab as Primary Treatment for Macular Edema Secondary to Central Retinal Vein Occlusion: Results of the Pan American Collaborative Retina Study Group at 24 Months,” Retina, Vol. 30, No. 7, 2010, pp. 1002-1011. http://dx.doi.org/10.1097/IAE.0b013e3181cea68d

[11] D. M. Brown, P. A. Campochiaro, R. P. Singh, et al., "Ranibizumab for Macular Edema Following Central Retinal Vein Occlusion: Six-Month Primary End Point Results of a Phase III Study,” Ophthalmology, Vol. 117, No. 6, 2010, pp. 1124-1133. http://dx.doi.org/10.1016/j.ophtha.2010.02.022
[12] A. E. Hoeh, M. Ruppenstein, T. Ach and S. Dithmar, "OCT Patterns of Macular Edema and Response to Bevacizumab Therapy in Retinal Vein Occlusion,” Graefe's Archive for Clinical and Experimental Ophthalmology, Vol. 248, No. 11, 2010, pp. 1567-1572. http://dx.doi.org/10.1016/j.ophtha.2010.02.022

[13] P. A. Campochiaro, G. Hafiz, R. Channa, et al., "Antagonism of Vascular Endothelial Growth Factor for Macular Edema Caused by Retinal Vein Occlusions: Two-Year Outcomes,” Ophthalmology, Vol. 117, No. 12, 2010, pp. 2387-2394.e1-5.

[14] H. Noma, H. Funatsu, T. Mimaura, et al., "Influence of Ischemia on Visual Function in Patients with Branch Retinal Vein Occlusion and Macular Edema," Clinical Ophthalmology, Vol. 5, 2011, pp. 679-685. http://dx.doi.org/10.2147/OPTH.S20527

[15] M. Tyberg, V. Ponjavic and M. Lovestam-Adrian, "Multifocal Electroretinogram (mfERG) in Patients with Diabetes Mellitus and an Enlarged Foveal Avascular Azone (FAZ),” Documenta Ophthalmologica, Vol. 117, No. 3, 2008, pp. 185-189. http://dx.doi.org/10.1007/s10633-008-9120-2

[16] Y. Yamaguchi, T. Otani and S. Kishi, "Serous Macular Detachment in Branch Retinal Vein Occlusion,” Retina, Vol. 26, No. 9, 2006, pp. 1029-1033. http://dx.doi.org/10.1097/01.iae.0000254893.94013.16

[17] A Tsujikawa, A Sakamoto, M Ota, et al., "Serous Retinal Detachment Associated with Retinal Vein Occlusion," American Journal of Ophthalmology, Vol. 149, No. 2, 2010, pp. 291.e5-301.e5.

[18] D. Finkelstein, "Ischemic Macular Edema. Recognition and Favorable Natural History in Branch Vein Occlusion," Archives of Ophthalmology, Vol. 110, No. 10, 1992, pp. 1427-1434. http://dx.doi.org/10.1001/archopht.1992.01080220089028 